\section{Dental education}

\section{ADEE independent review}

Sir, I write as a member of the Association for Dental Education in Europe (ADEE) project team, whose work was referred to in Shaun Sellars' recent article. ${ }^{1}$ As both a member of the team doing the research and a practising dentist, I am very grateful to the author for highlighting our vital review of professionalism. I agree with the vast majority of what Mr Sellars says, not least the argument put forward in the final paragraph, that for 'right touch' regulation, 'we need to understand what society as a whole, and not ourselves as individuals, deems to be appropriate. The only way to do this is to collect the information from dental professionals and the wider community?

It is important, though, for us to correct a factual inaccuracy contained within the article. Mr Sellars states that 'the GDC, in conjunction with Cardiff University, conducted a survey into professionalism in dentistry' and his final paragraph begins 'It could be argued that the GDC are not the right people to conduct this research'. I know that the GDC would agree with this argument, as indeed this is the very reason they commissioned the ADEE, as specialists in the field, to undertake the research independently.

Having successfully responded to the GDC's competitive tendering process, the ADEE conducted an extensive review of 'professionalism'. This four-part review included: a rapid evidence assessment of the global literature relating to dentistry and wider allied healthcare professions; scoping interviews with topic experts; focus groups with dentists, DCPs and members of the public; and a modified Delphi process (the 'survey' to which the author refers). The two-round Delphi survey was piloted with dental professionals and members of the public and had over 1,000 responses to round one and almost 700 to round two.

As Mr Sellars refers to in his article, there appeared at the time to be misunderstanding amongst some members of the profession about the motivation behind the survey, despite our inclusion of an introduction explaining the rationale and methodology of the research. I hope this clarification of the facts and the subsequent publication of the full review will alleviate any potential concerns.
The review is complete and due for publication in the near future. I am confident that it will prove to be an interesting read, and hope that further discussion will be generated on this vitally important subject.

J. Cowpe, Cardiff, UK

\section{Reference}

1. Sellars S. What is professional? Br Dent J 2020; 228: 571.

https://doi.org/10.1038/s41415-020-1738-9

\section{Antibacterial resistance}

\section{Start smart then focus}

Sir, the second audit of antimicrobial prescribing in secondary care has recently closed: a collaboration between the Association of Clinical Oral Microbiologists (ACOM), Association of Dental Hospitals $(\mathrm{ADH})$ and the English Surveillance Programme for Antimicrobial Utilisation and Resistance (ESPAUR). The month-long audit looked to build on the data collected in 2018 focusing on the therapeutic and prophylactic prescription practices in dental hospitals across the country. The importance of accuracy in appropriate prescription, in line with national guidance, and utilisation of the 'start smart then focus' protocol within our role as antimicrobial stewards cannot be understated. The Government's Antimicrobial Resistance Inquiry reports that antimicrobial resistant infections may claim tens of millions of lives per year, globally by 2050 if urgent action is not taken. Despite the data published by ESPAUR in 2018 showing a $25 \%$ reduction in antibiotic prescriptions by dentists, as a profession we are still responsible for $5.7 \%$ of all antibiotic prescriptions. Given the sizeable contribution to total antibiotic prescriptions that we as a profession are responsible for, we strongly urge practitioners in primary care to engage with the excellent toolkits and self-audit tools already published by Public Health England, Health Education and Improvement Wales and the Scottish Antimicrobial Prescribing Group.

Our collective efforts to ensure appropriate utilisation of antimicrobials to treat and prevent true infection, will help to protect the efficacy of existing treatments and buy the time required to develop new antibiotics and novel methods of treating infection.

R. Devine, Cardiff, UK https://doi.org/10.1038/S41415-020-1751-Z

\section{The history of dentistry}

\section{The Royal Dental Hospital}

Sir, may I, through your letter column, appeal to all ex-Royal Dental Hospital students and staff for support in an approach to Westminster Council for a Green Plaque to be placed on the outside of 32 Leicester Square, now a hotel, to commemorate the site of our Hospital from 1901 until its closure in 1985.

Some of you have already enthusiastically embraced this idea, but we would like the plaque to be 'owned' by as many ex-Royals as possible. For all of you with memories of the Royal and who wish our hospital to be recognised for what it stood for please send your details: name, email and years at the Royal, to brianwilliams14@btinternet.com. By so doing, you agree to be contacted by us for support in seeking approval from Westminster Council for a plaque. The greater our number the more likelihood of success.

The Royal Dental Hospital of London deserves recognition, not oblivion. It is up to all of us to ensure this doesn't happen. So please don't delay, email now. Thank you.

B. Williams, London, UK https://doi.org/10.1038/s41415-020-1739-8

\section{Ethics and law}

\section{Consensus, consent and DELPHI}

Sir, to achieve safe and effective dental care, there is a legal expectation that a competent practitioner obtains and maintains consent while keeping good clinical records. ${ }^{1}$ The Faculty of General Dental Practice (FGDP) has for two decades consistently developed three editions of their guideline: Clinical Examination and Record Keeping (CERK) acclaimed by independent experts to be of an excellent standard and widely recognised as a valuable dental resource. ${ }^{2}$

From 1 April 2020, the Department of Health and Social Care will implement a mandatory Systematised Nomenclature of Medicine Clinical Terms (SNOMED-CT) for NHS England dental practices. To achieve that aim an appointed Clinical Reference Group (CRG) used Delphi, a qualitatively ranked research protocol, circulating questionnaires to panels in four rounds until agreeing the items for inclusion in dental records. ${ }^{1,2}$ The CRG, its members taken from the GDC, NHSE and the FGDP, sought not to reinvent CERK, but to test its wider applicability, building on it, attaining 